Aus der Brustklinik, Upsala. (Dir.: Prof. Dr. R. Friberger.)

\title{
Ein Vergleich zwischen Lungentuberkulösen und Gesunden hinsichtlich tuberkulöser Exposition im Kindesalter.
}

Von

\section{Arvid Wallgren,}

Assistenzarzt.

Wie bekannt sein dürfte, war man ror einem Jahrzehnt fast allgemein der Ansicht, dass die Lungenschwindsucht beim erwachsenen Nenschen infolge einer direkten Primärinfektion der Lungen des Erwachsenen entstehe. Seit dem Jahre 1903, als v. Behring ${ }^{1}$ ) diese Auffassung der Phthise angriff und erklärte, es fehle jeder Beweis dafür, dass Tuberkelbazillen jemals durch Einatmen bei einem erwachsenen, zuvor tuberkelfreien Menschen Lungenkrankheit erzeugt bätten, ist diese Frage oft und viel umstritten worden. v. Behring meinte, dass die Entstehung der Lungenschwindsucht in den Kinderjahren zu suchen sei; er fasste sie als das Endstadium einer Infektion im Kindesalter auf.

v. Behring blieb jedoch nicht lange der einzige Vertreter der Ansicht, dass die Infektion im Kindesalter für die Entstehung der Lungenschwindsucht von grosser Bedeutung sei. Eine Reihe von Forschern haben sich dieser Theorie angeschlossen und sind warm dafür eingetreten; durch ihre mittelst verschiedener Untersuchungsmethoden gewonnenen Erfahrungen suchen sie die Richtigkeit derselben zu beweisen.

So haben z. B. Tuberkulinuntersuchungen an Kindern ergeben, dass im Pubertätsalter beinahe alle Fälle positiv reagiert haben, d. h. mit

1) Tuberkulosebekämpfung. Naturforscherversammlung. Kassel 1903. 
Tuberkulose infiziert waren. In Übereinstimmung hiermit haben nun verschiedene Sektionsstatistiken ergeben, dass fast alle Menschen in irgend einer Form Tuberkulose im Körper haben oder gehabt haben.

Durch Tierexperimente, die ron verschiedenen Forschern besonders von $R$ ömer $^{1}$ ) ausgeführt wurden, ist nachgewiesen worden, dass eine gelinde Infektion von Tuberkulose gegen eine erneute Infektion einen gewissen Schutz bieten kann. Hierauf nun sollte es zurückzuführen sein, dass die tuberkulöse Infektion bei Erwachsenen in den meisten Fällen keine Tuberkulose zur Folge hat. Die Infektion trifft ja fast immer ein früher in der Kindheit infiziertes, d. h. gewissermassen immunes Individuum. Nur wenn die neue Infektion massenhaft oder sehr virulent ist oder wenn die Immunität schwächer geworden ist, entsteht Lungenschwindsucht.

Eine neue Infektion, meint Römer, brauche nicht von aussen her zu kommen, sie käme vielmehr von innen, von dem primären Herd der Kinderjahre, d. h. sie wäre eine metastatische Autoinfektion. Die Ursache, weshalb nur gewisse Kinderinfektionen zu einer solchen Reinfektion der Erwachsenen und damit zu Lungenschwindsucht führen, sollte in der Quantität und Qualität der primären Infektion liegen. Ist diese intensiv, so ist die Bedingung für eine in späteren Jahren auftretende Lungenschwindsucht gegeben, ist sie dagegen weniger beträchtlich, so wird eine Immunität erworben, die einen, wenn auch nur begrenzten Schutz gegen spätere Tuberkulose bietet.

Sind nun diese Theorien richtig, so könnte man häufig intensive Infektionsgelegenheiten - Expositionen - während der Kindheit in der Anamnese Lungentuberkulöser nachweisen, während dies bei der Anamnese von Individuen mit gesunden Lungen nicht der Fall wäre, ein deutlicher Unterschied liesse sich hier nachweisen.

Es ist ja allgemein bekannt, wie häufig die Anamnese eines lungenschwindsüchtigen Patienten zeigt, dass Lungenschwindsucht in der Familie des Kranken vorgekommen ist. Damit ist jedoch weder gesagt, dass die Infektion gerade von dem tuberkulösen Verwandten ïbertragen worden sein muss, noch dass der Kranke mehr der Ansteckungsgefahr ausgesetzt worden ist, als rielleicht ein anderer, in dessen Familie keine Tuberkulose vorkam. Um annelımen zu können, dass eine Exposition für Tuberkulose stattgefunden hat, muss nachgewiesen werden, dass eine Beriihrung zwischen der eventuellen tuberkulösen Ansteckungsquelle und dem Infizierten erfolgt ist.

Welch ein Unterschied zwischen tuberkulöser Exposition und tuberkulöser Heredität - wenn man dieses Wort verwenden darf -

1) Brauers Beiträge Bd. XI, XIII, XVII und XXII. 
bestehen $\mathrm{kann}$, ist aus folgenden, von Tillis $\mathrm{ch}^{\mathbf{1}}$ ) gefundenen Zahlen zu ersehen. Er hat die Anamnese von 841 Lungenschwindsüchtigen (398 Männern und 443 Frauen) mit Rücksicht auf das Vorkommen einer Exposition für Tuberkulose während der Kindheit bearbeitet. Nur in 168 Fällen $=20 \%(17,3 \%$ Yänner und $22,3 \%$ Frauen $)$ konnte er nachweisen, dass der Kranke im Kindesalter der Tuberkulose ausgesetzt war. Dagegen fand sich in 385 Fällen $=45,8 \%$ bei Eltern und Geschwistern Tuberkulose. Die Ursache dieses grossen Unterschiedes zwischen der Exposition eines Kindes und der Familientuberkulose dürfte teils darin zu suchen sein, dass eine grosse Anzahl von tuberkulösen Krankheitsfällen in der Familie des Patienten erst vorgekommen sind, nachdem er die Kinderjahre hinter sich hatte; teils darin, dass bei den Geschwistern die Tuberkulose erst aufgetreten ist, nachdem sie das Elternhaus verlassen hatten, der Patient demnach also nicht mit ihnen in Berührung gewesen war. Ferner dürfte es leichter sein, eine positive Familienanamnese eines Lungenschwindsiichtigen als eine positive Exposition in der Kindheit nachzuweisen.

Es ist natürlich eine heikle Sache aus Angaben in der Anamnese betreffs der Exposition in der Kindheit sichere Schlüsse zu ziehen. In wie hohem Grade anamnestische Auskünfte irreführen können, weiss jeder Arzt aus eigener Erfahrung. Jede Aufnahme einer wirklich zuverlässigen Anamnese stellt grosse Anforderungen sowohl an die Tüchtigkeit als auch an die Gerluld und Zeit des Arztes. Es ist selbstverständlich, dass die Art und Weise, sowie die Gründlichkeit beim Ausfragen eine grosse Rolle spielen. Das Ergebnis der Anamnese ist jedoch auch zu einem gewissen Grad von dem intellektuellen Niveau des Ausgefragten abhängig, ferner von seiner Fähigkeit, erlebte Momente im Gedächtnis festzuhalten und besonders schliesslich von der Affektsbetonung, die die einzelnen früheren Ereignisse begleitet hat. Diese wiederum dürfte auf einer derzeitigen, allgemeinen Kenntnis des Wesens und der Ursache der Krankheit, besonders der Ansteckungsgefahr, beruhen (Bruch und Steinberg $\left.{ }^{2}\right)$ ). Ferner muss man, wie auch

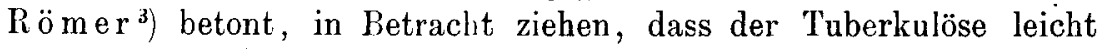
dazu neigt, seine Krankheit mit einer naheliegenden Ursache in $\mathrm{Zu}-$ sammenhang zu setzen, was ja leicht erklärlich ist, da jeder Kranke wohl lieber an einer akuten als an einer lange vorhandenen chronischen Krankheit leiden will.

1) Alb. Tillisch: Omkring spöry maalet exogen eller endogen reinfection ved lungetuberkulose. Medicinsk Revue 31 Aarg. M. 5.

$\left.{ }^{2}\right) \mathrm{Bruch}$ und Steinberg: Die Verbreitung der Lungentuberkulose in Breslauer Familien, Wohnungen und Werkstätten. Zeitschrift für Hygiene und Infektionskrankheiten. Bd. 71.

3) Brauers Beiträge Bd. XVII. 
In der Literatur finden sich mehrere statistisch anamnestische Untersuchungen, die alle unternommen worden sind, um die Bedeutung der Exposition bzw. der primären Kindheitsinfektion für eine in späteren Lebensjahren ausgebrochene Lungentuberkulose zu erforschen.

So haben Ritter und Vehling ${ }^{1}$ ) im Sanatorium Edmundstal anamnestische Auskünfte von 200 mit Lungentuberkulose behafteten Patienten (100 Männern und 100 Frauen) mit Rücksicht auf das eventuelle Vorkommen einer tuberkulösen Infektion in der Kindheit bearbeitet. Als Ausdruck einer solchen Infektion betrachten diese Verfasser chronische Drüsenanschwellungen, Knochen- und Gelenkkrankheiten, chronische Katarrhe in den Atmungsorganen, chronischen Eiterfluss aus den Ohren; ferner rechnen sie auch zu den Infizierten die seit der Kindheit schwachen, kränklichen, sowie blutarmen Individuen, besonders wenn sich diese Schwäche in unregelmässigem Schulbesuch, in Unfähigkeit für körperliche Arbeit in späteren Jahren, in Militäruntauglichkeit usw. gezeigt hat. Die Forscher gehen jedoch zu weit darin, alle diese Schwächesymptome als einen Ausdruck der Tuberkulose zu betrachten. Aller Wahrscheinlichkeit nach würden sie in ihrer Praxis nicht Tuberkulose bei solchen Kindern festgestellt haben, an denen einige dieser Symptome vorhanden waren.

Von den 200 Patienten waren 41 Männer und 54 Frauen in der Kindheit mit Tuberkulose infiziert worden und hatten mit tuberkulösen Eltern und Geschwistern zusammengelebt. 37 Männer und 33 Fraven waren in der Kindheit infiziert worden, ohne dass bei Eltern oder Geschwistern Tuberkulose vorgekommen war. 8 Männer und 6 Frauen sind der Ansteckung von Eltern oder Geschwistern ausgesetzt gewesen, haben jedoch keine Symptome von tuberkulöser Infektion gezeigt. Schliesslich sind 14 Männer und 7 Frauen weder einer Ansteckung ausgesetzt gewesen noch haben sie in ihrer Kindheit Symptome von Tuberkulose gezeigt. Tuberkulöse Infektion in der Kindheit ist also bei 165 Fällen, ron welchen 87 Frauen und 78 Männer waren, aufgetreten. 49 Männer und 60 Frauen sind der Ansteckung von Eltern oder Geschwistern ausgesetzt gewesen.

v. Hellens ${ }^{2}$ ) hat aus dem Sanatorium Nummela ein Material ron 1400 Fällen mit Lungentuberkulose gesammelt und bei einer ähnlichen Untersuchung gefunden, dass nur in $16,29 \%$ aller Fälle weder das Vorhandensein von Tuberkulosesymptomen in der Kindheit noch Tuber-

1) Ritter und Vehling: Berl. klin. Wochenschr. 1909, S. 1924.

2) Finska läkaresällskapets handlingar. Bd. LV. Nr. 5. 
kulose bei den nächsten Angehörigen konstatiert werden konnte. Er hat jedoch nicht die Ansteckungsmöglichkeiten in der Kindheit angegeben, sondern nur das Vorhandensein von Tuberkulose in der Familie, und zwar bei Eltern und Geschwistern. Seine Arbeit zeigt also, was man die Heredität der Lungenschwindsüchtigen für Tuberkulose nennen könnte, nicht aber ihre Exposition für Tuberkulose in der Kindheit.

Bruch und Steinberg ${ }^{1}$ ) haben nach Fragebogen, die sie durch die Gemeindepflegerin in Breslau ausgehen liessen, und nach eigenen Untersuchungen an 300 Fällen mit Lungentuberkulose die Exposition für Tuberkulose und die Krankheitssymptome in der Kindheit genau studiert. Sichere Exposition in der Kindheit haben sie in 29,5\% gefunden, in 38,9\% entweder in der Kindheit oder im späteren Alter und in $31,6 \%$ im späteren Alter.

Freymuth${ }^{2}$ ) hat die Anamnese von 510 Phthisikern aufgenommen, seiner Meinung nach fanden sich keine Beweise dafür, dass der grösste Teil oder auch nur ein erheblicher Teil schon als Kind tuberkulös infiziert worden war. Seine Anamnesen sind jedoch nicht mit besonderer Rücksicht auf die Möglichkeit einer Exposition im Kindesalter aufgenommen und deshalb will ich hier nicht näher darauf eingehen.

Viel zitiert worden sind Hillen bergs ${ }^{3}$ ) Untersuchungen. Unter 1000 Fällen mit Lungentuberkulose fand er in $41 \%$ eine sichere Infektionsquelle, aber nur in 14,3\% war die Ansteckung in der Kindheit erfolgt. Deshalb ist er der Ansicht, dass die Lehre, die Lungenschwindsucht bei Erwachsenen sei nur ein Produkt einer metastatischen Autoinfektion von einem infantilen Herd, unhaltbar sei. Hillenbergs Untersuchungen weisen jedoch grosse Fehlerquellen auf. Der grössere Teil seiner Fälle bestand z. B. aus bereits Gestorbenen, einige waren sogar schon mehrere Jahre tot. Über diese nun sichere Auskünfte betreffs Exposition für Tuberkulose zu erhalten, ist natürlich in den meisten Fällen unmöglich. Gewisse Schlüsse ${ }^{4}$ ), die er gezogen hat, sind jedoch von Interesse. Er meint, dass die Infektionen, wo die Quelle für die Infektion nicht bekannt ist, gutartiger Natur sind und bei höherem Alter nicht zu manifester Tuberkulose führen, sondern vielmehr immunisierend wirken.

Nikolski ${ }^{5}$ ) hat eine Anamneseuntersuchung über das Auftreten der Tuberkulose innerhalb der Familie an 113 Patienten ausgeführt,

1) Zeitschrift für Hygiene und Infektionskrankheiten. Bd. 71 .

2) Beiträge zur Klinik der Tuberkulose. Bd. 33 .

3) Zeitschrift für Hygiene und Infektionskrankheiten. Bd. 65.

4) Tuberkulosis. 1911.

5) Zeitschrift für Tuberkulose Bd. 7 . 
von diesen hatten 58 Lungentuberkulose, die anderen 55 waren nicht tuberkelkrank. Er fand Familientuberkulose bei den Lungentuberkulösen in $87,90 \%$, bei den Nichttuberkulösen in $34,5 \%$. Die Eltern waren in $50 \%$ bzw. $11 \%$ tuberkelkrank, und deshalb nimmt Nikols ki an, dass die tuberkulösen Patienten fünfmal mehr als die nichttuberkulösen Individuen der Infektion ausgesetzt worden sind. Angesichts des Unterschiedes, der sich zwischen "tuberkulöser Heredität“ und tuberkulöser Exposition findet, und auf den auch ich aufmerksam gemacht habe, muss man diesen Schlusssatz Nikolsk is wahrscheinlich als nicht völlig mit der Wirklichkeit übereinstimmend ansehen. Könnte man Heredität und Exposition als gleichwertig betrachten, so wäre ja $\mathrm{Nikolskis} \mathrm{Untersuchung} \mathrm{als} \mathrm{eine} \mathrm{Stütze} \mathrm{für} \mathrm{die} \mathrm{Röm} \mathrm{ersche} \mathrm{Theorie}$ von der metastatischen Autoinfektion anzusehen.

Betreffs der jetzt besprochenen Untersuchungen kann zu allererst bemerkt werden, dass die extrafamiliäre Exposition im Kindesalter oft allzuwenig beachtet worden ist. Des weiteren hat man oft zwischen .tuberkulöser Heredität" und tuberkulöser intrafamiliärer Exposition keinen genügenden Unterschied gemacht. Die Schlüsse divergieren auch merklich.

Bei dieser Sachlage habe ich, um das Verhalten der tuberkulösen Infektion in der Kindheit zur Lungenschwindsucht in späteren Jahren zu beleuchten mit Rücksicht auf das Vorkommen sicher nachweisbarer Kindheitsexposition sowohl innerhalb als ausserhalb der Familie einen Vergleich zwischen Individuen mit Lungentuberkulose und solchen mit gesunden Lungen angestellt. Mit der Familie werden hier sowohl Eltern und Geschwister des Betreffenden als auch die Grosseltern und die Geschwister der Eltern gemeint.

Mein Material von Lungentuberkulösen bestand aus 100 Patienten, die im Jahr 1914 in der Brustklinik des Akademischen Krankenhauses zu Upsala behandelt wurden. Nur durchaus sichere Fälle von Lungentuberkulose sind aufgenommen worden, und von diesen nur die, die unter 40 Jahren waren. Was die Zuverlässigkeit in den Angaben über die Kindheit anbetrifft, so wird diese natürlich mit zunehmenden Jahren geringer. Bei einem Patienten, der über 40 . Jahre alt ist, ist es selten, sichere Anskünfte über die Kinderjahre zu erhalten. Die grösste Anzahl der Patienten stammt aus mehr oder weniger armen Häusern, wo die Ansteckungsgefahr sicher grösser war, als bei $\mathrm{Pa}$ tienten aus den oberen Klassen, ein Umstand, der jedoch vielleicht. dadurch aufgewogen wird, dass man von ersteren nicht ebenso genaue und zuverlässige Auskünfte über die eigene Person verlangen kann, als es bei letzteren der Fall ist. Die Auskünfte in der Anamnese sind, was die Exposition in den Kinderjahren angeht, soweit es mög- 
lich war, ausser vom Patienten selbst, auch von den nächsten Angehörigen desselben eingezogen worden. Oft ist eine vom Patienten selbst abgegebene negative Anamnese nach Ausfragen z. B. von der Mutter des Betreffenden in eine positive verwandelt worden, da man ron dieser Auskünfte über die Ansteckungsquelle während der Kinderjahre erhalten hatte.

Die Anamnesen, die zum Teil von dem früheren Assistenten, Dr. Erik Ericsson und zum grössten Teil von mir selbst herrühren, sind alle nach einem einheitlichen Plan und mit besonderer Rücksicht auf eventuelle Expositionen in der Kindheit aufgenommen worden. Nur in den Fällen, wo man mit Sicherheit nachweisen konnte, dass der Patient während seiner Kindheit wirklich einer intimen Berührung mit einem hustenden Lungenkranken ausgesetzt war, habe ich geglaubt annehmen zu dürfen, dass eine Exposition für Tuberkulose vorgelegen hat. Als eine Ansteckungsquelle, d. h. als hustende Schwind. süchtige betrachtete man solche Personen, die an langwierigen Hustenkrankheiten gestorben sind, die an langwierigem Husten mit Lungenblutungen gelitten haben, oder von denen die Patienten selbst angeben, dass sie an Lungenschwindsucht, Blutsturz oder Kehlkopfschwindsucht gelitten haben oder daran gestorben sind. Nun könnte die Möglichkeit vorhanden sein, dass dieser oder jener der Hustenden, der als eventuelle Ansteckungsquelle angesehen wurde, nicht an Lungenschwindsucht, sondern an einer anderen chronischen Hustenkrankheit z. B. Emphysembronchitis, Bronchiektasien etc. gelitten hat: ich habe mich jedoch so viel wie möglich bemüht, diese Fehlerquellen unter meinem Material zu vermeiden.

Einen ganz sicheren Zeitpunkt für die Infektion festzustellen stösst auf grosse Schwierigkeiten. Man bekommt z. B. die Auskunft, die Mutter sei an der Schwindsucht gestorben, als der Patient 14 Jahre alt war, seit etwa 5 Jahren vor ihrem Tode hätte sie gehustet. Das Kind ist demnach zwischen dem 9. und 14. Lebensjahre der Ansteckung von seiten der Mutter ausgesetzt gewesen, in welchem Jahre nun die Infektion eingetreten ist, ist natürlich schwieriger $\mathrm{zu}$ bestimmen. Ich habe angenommen, dass die zu einer Infektion führende Exposition zu der Zeit stattgefunden hat, wo das Kind zum ersten Male der Ansteckung ausgesetzt gewesen ist, in diesem Falle also im 9. Lebensjahre.

Beim Aufnehmen der Anamnese betreffs der Exposition habe ich mich zuerst danach erkundigt, ob ein Fall von Lungentuberkulose in der Familie des Patienten vorgekommen war. Wurde diese Frage positiv beantwortet, so habe ich nachgeforscht, wann der Lungenschwindsüchtige zu husten angefangen habe, und ob der Patient mit 
ihm in Berührung gewesen war, und wenn dies der Fall war, wann. Ferner habe ich den Patienten nach allen Personen ausgefragt, die sich zu einem bestimmten Zeitpunkt während seiner Kindheit in seinem Elternhause aufgehalten haben, wer diese Personen waren, und ob einer von ihnen gehustet oder Tuberkulose gehabt hat. Hatte sich eine hustende Person im Elternhaus des Patienten aufgehalten, so habe ich zu erkunden gesucht, ob dieser Hustende an Lungentuberkulose gelitten hat. Auf gleiche Weise ist der Patient über seine Nachbarn, seine Spiel- und Schulkameraden etc. ausgefragt worden. Ich habe mich erkundigt, wann der Patient sein Elternhaus verlassen hat, wo er sich dann aufgehalten hat, ob sich hustende Personen in seiner Umgebung befunden haben usw.

Die Personen mit gesunden Lungen, an denen ich ähnliche anamnestische Untersuchungen über das Vorkommen einer Exposition für Tuberkulose während der Kindheit unternommen habe, waren 100 Personen bis zum 40. Lebensjahre; teils gehörten sie dem Krankenhauspersonal an, teils den Patienten der Poliklinik und teils den jungen Medizinern, die im Krankenhause arbeiteten. Letztere hatten den Auftrag, sich während der Ferien bei ihren Angehörigen über eine Infektionsmöglichkeit in der Kindheit zu erkundigen und bei ihrer Rückkehr schriftliche Angaben hierüber zu machen.

Betreffs der Möglichkeit eines Vergleiches zwischen den anamnestischen Angaben der Lungenschwindsüchtigen mit entsprechenden Angaben der Gesunden, mag darauf aufmerksam gemacht werden, dass ein lungenschwindsüchtiger Patient in seiner Umgebung dem Auftreten der Krankheit, an der auch er leidet, mehr Beachtung schenkt als ein Gesunder. Dieses könnte nun dazu beitragen, die Frequenz des positiven anamnestischen Befundes jener Kategorie zu erhöhen. Es muss jedoch andererseits darauf hingewiesen werden, dass eine grosse Anzahl der in Frage kommenden Gesunden mehr als der Durchschnitt der übrigen dazu fähig waren, vollständige Angaben zu liefern. Besonders gilt dies von den unter das Material aufgenommenen Medizinern und den 38 Krankenpflegerinnen.

Es fragt sich ferner, ob die beiden Kategorien - die Lungenkranken und die Gesunden - gleich situierten Klassen angehört haben, so dass ein Vergleich möglich ist. Unter der ärmeren Bevölkerung bieten sich ja grössere Möglichkeiten für eine Exposition als unter den reichen. Ungefähr die Hälfte meines Materials von Gesunden bestand aus solchen Personen, die derselben Klasse angehörten, wie meine lungenkranken Patienten. Die andere Hälfte, die Pflegerinnen und die Mediziner, hat sicher den grössten Teil ihrer Kindheit in besser situierten Familien zugebracht als diese. Dass dieser soziale Unter- 
schied doch nicht allzuviel zu sagen hat, geht daraus hervor, dass nicht weniger als 6 von den 20 Medizinern Angaben über Exposition in der Kindheit gemacht haben.

Alle als mit gesunden Lungen Angegebenen sind einer Untersuchung der Lungen unterzogen worden. Nur diejenigen, deren Lungen bei einer gewöhnlichen physikalischen Untersuchung ein völlig physiologisches Verhalten zeigten, und die subjektiv keine Lungensymptome gehabt haben, sind $\mathrm{zu}$ der Gruppe der Gesunden gerechnet worden. Ergab die physikalische Untersuchung zweifelhafte Resultate, so wurde eine Röntgenuntersuchung zu Hilfe genommen. Von den 100 Personen mit gesunden Lungen bestand die eine Hälfte aus Männern, die andere aus Frauen, von den Lungenkranken waren 46 Männer und 54 Frauen.

Tabelle 1.

Lungentuberkulöse.

Die tuberkulöse Exposition geschah zwischen

\begin{tabular}{|c|c|c|c|c|c|c|c|}
\hline & $0-5$ & Jahren & $5-10$ & Jabren & $10-15$ & Jahren & \\
\hline Infektionsquelle & Männer & Frauen & Männer & Frauen & Männer & Frauen & Summa \\
\hline Eiltern & 7 & 4 & 1 & 3 & 7 & 5 & 27 \\
\hline Geschwister & 1 & - & - & 1 & 4 & - & 6 \\
\hline Andere Verwandte & 1 & 1 & 2 & 4 & 1 & 2 & 11 \\
\hline \multicolumn{8}{|c|}{ Ausserhalb der Familie } \\
\hline Stehende & 1 & - & 1 & 1 & 2 & 2 & 7 \\
\hline & 10 & 5 & 4 & 9 & 14 & $y$ & 51 \\
\hline
\end{tabular}

Tabelle 2.

Individuen mit gesunden Lungen.

Die tuberkulöse Exposition geschah zwischen

$$
\text { 0-5 Jahren 5-10 Jahren 10-15 Jahren }
$$

Infektionsquelle Männer Frauen Männer Frauen Männer Frauen Summa

Eltern

Geschwister

Andere Verwandte

Ausserhalb der Familie

Stehende

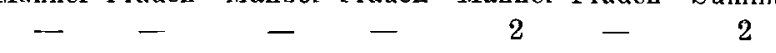

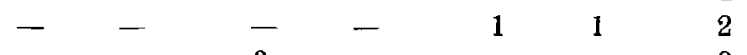

$1 \quad-$

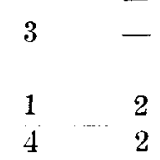

- -3

Betreffs obiger Tabelien, in denen die Ergebnisse vorliegender Untersuchung zusammengefasst worden sind, unag zuerst auf ein paar Details aufmerksam gemacht werden. Es ist in die Augen fallend, dass die Männer häufiger eine positive Anamnese als die Frauen abgegeben haben; im Gegensatz zu dem, was andere Forscher gefunden haben und was sie dahin erklären, dass die von Männern abgegebenen anamnestischen Angaben sehr oft unvollständiger sind als die 
von den Frauen. Von den Lungenkranken haben $60,9 \%$ der Männer und $42,6 \%$ der Frauen, von den Gesunden $20 \%$ der Männer und $6 \%$ der Frauen über Exposition für Tuberkulose in der Kindheit Angaben gemacht.

Die Ansteckungsquellen waren unter den Lungenkranken in $27 \mathrm{Fäl-}$ len die Eltern, in 6 Fällen die Geschwister, in 11 Fällen andere Angehörige und in 7 Fällen ausserhalb der Familie stehende Personen. Die Individuen mit gesunden Lungen waren in 2 Fällen von den Eltern, in 2 Fällen von den Geschwistern, in 3 Fällen von anderen Angehörigen und in 6 Fällen von Lungenkranken ausserhalb der Familie einer Exposition ausgesetzt. Auffallend ist die grosse Expositionszahl durch ausserhalb der Familie Stehende sowohl bei den Lungenkranken als bei den Gesunden, zusammen nicht weniger als 13 Fälle. Ich habe keine Angaben über diese extrafamiliäre Exposition in der Kindheit von anderer Seite gefunden, was wahrscheinlich darauf zurückzuführen ist, dass diese Möglichkeit nicht hinreichend beachtet worden ist. Es kann daher vielleicht von Interesse sein, diese Fälle als Beispiele solcher Exposition näher zu beschreiben:

1. A. H., 23 Jahre alt, lungentuberkalös. Nummer des Krankenjournals 58: 1914. Die Mutter ist an Krebs gestorben; der Vater an einer unbekannten Krankheit, als Pat. ein kleines Kind war. Ein Bruder ist gesund. Als Pat. 14 Jatre alt war, wobnte er in demselben Hause, wie ein lungenschwindsiichtiger Mann in mittleren Jahren, mit dem er zuweilen in Berührung war.

2. A. A., 36 Jahre alt, lungentuberkulös. 102: 1914. Die Elteru starben an einer unbekannten Krankheit, als Pat. drei Jahre alt war. Drei Geschwister sind gesund. Nach dem Tode der Eltern kam Pat. in eine Versorgungsanstalt, wo er mit einem hustenden Manne zusammengewohnt und oft aus demselben Glase wie dieser getrunken Laben soll.

3. M. N., 28 Jahre alt, Iungentuberkulös. 81: 1914. Die Eltern sind am Leben. Die Mutter hat ungefähr seit dem 10. Lebensjahre des Patienten gehustet. Der Arzt hat sie jedoch nach wiederholten Untersuchungen für tuberkulosefrei erklärt. Zwei Geschwister sind gesund. Pat. war während seiner ganzen Kindheit täglich mit einem Mädchen zusammen, das viel gehustet hat und an Schwindsucht gestorben ist.

4. A. S., 29 Jahre alt, lungentuberkulös. 117: 1914. Eltern und drei Geschwister sind gesund. Als der Patient den Konfirmandenunterricht besuchte, wohnte er in einer schwindsüchtigen Familie.

5. N. J., 20 Jahre alt, lungentuberkulös. 118: 1914. Eitern und neun Geschwister sind gesund. Als Pat. die Schule besuchte, hatte er einen hustenden Schalkameraden, der später an Lungenschwindsucht gestorben ist.

6. F. K., 36 Jahre alt, lungentuberkulös. 132: 1914. In der Familie ist keine Tuberkulose. Ein Schulkamerad, mit dem Pat. verkehrt hat, war lungenkrank.

7. E. F., 17 Jahre alt, lungentuberkulös. 141: 1914. Eltern und vier Geschwister sind gesund. Ein Bruder ist an Lungenentzündung gestorben. Als Pat. 
15 Jahre alt war, wohnte er ein Jahr lang in einer lungenechwindsüchtigen Familie. Eine andere Infektionsquelle ist nicht bekannt.

8. 'T. O., cand. med., gesund. In der Familie ist keine Tuberkulose. Ein Schüler in seiner Klasse starb an Schwindsucht, als T. O. 12 Jahre alt war.

9. A. T., 21 Jahre alt, cand. med., gesund. Eltern und Geschwister sind gesund. Keine Jungenschwindsucht in der Familie. Als A. T. 5-6 Jahre alt war, hielt sich eine lungenkranke Person in der Familie auf.

10. A. B., 17 Jahre alt, Gärtnerlehrling, lungengesund. In der Familie ist keine Schwindsucht vorgekommen. Als A. B. 14 Jahre alt war, besuchte er öfters einen alten hustenden Mann, der wahrscheinlich lungenkrank war.

11. J. P., Schüler, lungengesund. Pat. war zwiscìen seinem 5.-15. Lebensjahr häufig mit einem Mädchen zusammen, das $17 \mathrm{Jahr}$ alt an einer tuberkulösen Meningitis gestorben ist, und in dessen Elternhaus mehrere der Familienmitglieder an Tuberkulose gelitten und gehustet haben sollen. Pat. war oft in dieser Familie.

12. A. A., 33 Jahre alt, Krankenpflegerin, gesund. Die Eitern sind gesund. Pat. bat elf Geschwister gehabt, von denen sieben tot sind. Eins derselben starb an einer Darmkrankheit, mehrere an akuten Lungenkrankheiten nach Masern; eins infolge von Schlag", eins an unbekannter Krankheit. Im Jahre 1889 pflegte die Mutter eine lungenkranke Frau, und bekam von dieser eine Decke, die nicht desinfiziert worden war. Dasselbe Jahr starb ein elf Monat altes Kind an Meningitis. 1839-1894 war A. A. für 'Tuberkulose ansgesetzt. Sie half zu der Zeit bei der Pflege eines langenkranken Mädchens, das später an Tuberkulose starb. Eine Schwester, 1884 geboren, hustete als Kind viel und hat jetzt eine Spitzenaffektion. Ein Bruder, 1887 geboren, hat Lungenbluten und Pleuritis gehabt.

13. K. P., 25 Jahre alt, cand. med., gesund. Fitern und Geschwister leben, alle sind vollkommen gesund. Kein Fall von Lungenkrankheit in der Familie. Im Alter von $2 \% / 2$ bis zu 15 Jahren war Pat.* oft in einer Familie, wo sich eine lungenkranke Person aufhielt. Diese kannte die Ansteckungsgefahr und benutzte ein Taschenspuckgefäss, beobachtete auch andere Vorsichtsmassregeln, um einer Ansteckung vorzubeugen. Als Pat. sechs Jahre alt war, kam eine völlig gesunde Erzieherin ins Haus. Nach $2^{1 / 2}$ Jahren bekamen alle Geschwister Keuchhusten, auch die Erzieherin fing zu husten an, wurde nach zwei Monaten vom Arzte untersucht, der gallopierende Schwindsucht feststellte, kurze Zeit darauf starb sie. Während dieser Zejt war K. P. jedoch in keiner Berührung mit ihr gewesen.

Was nun die Hauptfrage selbst angeht, so geht aus den Tabellen hervor, dass sich eine tuberkulöse Exposition in der Kindheit in 51\% der Fälle unter den Lungenkranken, aber nur in $13 \%$ unter den Gesunden nachweisen liess. Es versteht sich von selbst, dass es sich hinsichtlich beider Kategorien um Minimumzahlen handelt; für die Patienten sind natürlich öfter Expositionen für Tuberkulose, als nachgewiesen werden konnte, vorhanden gewesen; die hier angegebenen können jedenfalls für so gut wie sicher betrachtet werden.

Der Unterschied zwischen den Gesunden und den Kranken ist auffallend; das Verhältnis ist ungefähr wie 1:4. Bietet nun auch eine Untersuchung wie diese in mancher Hinsicht vielleicht. nicht 
völlige Sicherheit, so dürfte jedoch ein so ausgeprägtes Resultat nicht ausschliesslich dem Zufall zuzuschreiben sein. Im Gegenteil scheint man nach vorliegender Untersuchung berechtigt $\mathrm{zu}$ sein, auf das Vorkommen eines Zusammenhanges zwischen der tuberkulösen Exposition während derKindheitundder Lungenschwindsucht im späteren Alter zu schliessen. Worin nun dieser Zusammenhang besteht, erläutert die Untersuchung nicht näher. Es ist jedoch wohl ausgeschlossen, mit Hinsicht darauf, was oben über die Beschaffenheit des Materials gesagt wurde, sich diesen Zusammenhang als einen rein äusseren vorzustellen, z. B. hygienische und soziale Verhältnisse, das Gebundensein an eine gewisse Umgebung usw. Man möchte vielmehr den Zusammenhang in mehr oder weniger strengem Anschluss an die von $\mathrm{Röm}$ er angegebene Theorie deuten.

Wie schon erwähnt, meint $\mathrm{R} \ddot{\mathrm{m}} \mathrm{er}$, dass die besonders intensiven Expositionen für Tuberkulose in der Kindheit zur Lungenschwindsucht in späteren Jahren führen. Pollak ${ }^{1}$ ) hat in einer Untersuchung von Kindern zu finden geglaubt, dass die Periode der Kindheit, wo die Infektion geschieht, für ihre Folgen wenigstens für die nächsten Jahre bestimmend ist. Geschieht die Infektion während der ersten vier Lebensjahre, so führt sie zu weit ernsteren, unmittelbaren Phänomenen als wenn sie später geschieht. Mit Rücksicht hierauf ist es von grossem Interesse, dass ich eine Exposition vor dem 5. Lebensjahre in $15 \%$ der Fälle unter den Schwindsüchtigen habe nachweisen können, dagegen aber nur in $1 \%$ unter den Gesunden. Wollte man nun hieraus einen Schlusssatz ziehen, so dürfte es der sein, dass eine frühe Infektion während der Kindheit auch fürdie spätere Zukunft verhängnisvoller ist als eine Infektion, die geschieht, wenn das Kind etwas älter ist.

\section{Zusammenfassung.}

Die Untersuchung besteht aus einem Vergleich zwischen Angaben hinsichtlich tuberkulöser Exposition in der Kindheit von 100 Lungenschwindsüchtigen und 100 Personen ohne Schwindsucht. Alle Untersuchten waren unter $40 \mathrm{Jahren}$.

Tuberkulöse Exposition fand sich in $51 \%$ bei den Lungenkranken, in $13 \%$ bei den Gesunden; eine Exposition vor dem 5 . Jahre in $15 \%$ der Lungenkranken und nur in $1 \%$ der Gesunden.

3) Brauers Beiträge. Bd. XIX. 